\title{
Updated pulsation models for BL Herculis stars
}

\author{
M. Marconi ${ }^{1}$ and M. Di Criscienzo ${ }^{1,2}$ \\ 1 INAF - Osservatorio Astronomico di Capodimonte, via Moiariello 16, 0080131 Naples, Italy \\ e-mail: dicrisci@na.astro.it \\ 2 Universitá di Roma "Tor Vergata”, via della Ricerca Scientifica 1, Rome, Italy
}

Received 26 October 2006 / Accepted 2 January 2007

\section{ABSTRACT}

\begin{abstract}
Context. Population II pulsating variables play a relevant role both as distance indicators and as tracers of the properties of old stellar populations.

Aims. In this paper we present an updated and homogeneous pulsational scenario for a wide range of stellar parameters typical of BL Her stars, i.e., Population II Cepheids with periods shorter than 8 days.

Methods. For this purpose, we adopt a nonlinear convective hydrodinamical code to evaluate the stability and full amplitude behavior of an extensive set of BL Her pulsation models. Various assumptions of mass, luminosity, and metallicity consistent with the most recent evolutionary prescriptions, are adopted.

Results. We obtain the theoretical instability strip for both fundamental and first overtone pulsators and present a detailed atlas of light/radial-velocity curves. Some relations for the boundaries of the instability strip and for the dependence of the absolute magnitude on period, mass, and color, as well as the fundamental period-amplitude relations are derived. Finally, we provide the theoretical period-radius relation for BL Her and find that it is in an excellent agreement with the empirical relation by Burki \& Meylan (1986, A\&A, 159, 255) and is consistent with the one holding for shorter periods for RR Lyrae stars.
\end{abstract}

Key words. stars: population II - stars: horizontal-branch - stars: variables: general

\section{Introduction}

The BL Her variables form a small but interesting group of radially pulsating Population II stars with period $P$ in the range 0.8-3 days. They are observed in globular clusters with few RR Lyrae stars and blue horizontal branch (HB) morphology and they are brighter than RR Lyrae but fainter than Anomalous Cepheids, for a fixed period. They are currently interpreted as central He-burning low mass $\left(M \leq 0.6 M_{\odot}\right)$ stars which, after their main core He-burning phase spent in the blue side of the Zero Age Horizontal Branch (ZAHB), evolve toward the Asymptotic Giant Branch (AGB) crossing the instability strip at luminosities higher than the RR Lyrae level (see Gingold 1985; Bono et al. 1997a; Caputo 1998).

The properties of these objects have recently been reviewed by Wallerstein (2002) and Sandage \& Tammann (2006). From the theoretical point of view, Buchler \& Moskalik (1992) and Buchler \& Buchler (1994) presented a pulsational study of models with $P \leq 3$ days based on a linear and nonlinear radiative analysis. These authors found evidence for a resonance between the fundamental mode and the second overtone, similar to the one often invoked as the explanation of the Hertzsprung progression phenomenon in Classical Cepheids (see Bono et al. 2000, for a detailed discussion). Buchler \& Buchler (1994) also evaluated the pulsation properties of first overtone (FO) models and found that the first overtone blue edge (FOBE) was very close $(\leq 100 \mathrm{~K})$ to the fundamental $(\mathrm{F})$ one, producing a very narrow regime of FO-only pulsation. Moreover, FO models were found to show very low pulsation amplitudes, but it seemed to be hard to discriminate the pulsation mode on the basis of their Fourier coefficients. By relying on a nonlinear convective pulsation analysis, Bono et al. (1997a) found a good agreement between the predicted and the observed BL Her instability strip boundaries and suggested that these pulsators are pulsating in the $\mathrm{F}$ mode with a typical mass of $0.52-0.59 M_{\odot}$. Moreover, these authors predicted a period-luminosity-amplitude (PLA) relation in the $B$ band.

However, the Bono et al. (1997a) nonlinear convective approach was limited to a quite restricted range of stellar parameters and adopted an old input physics both in the evolutionary and the pulsational computations (see Bono \& Stellingwerf 1994; and Bono et al. 1997b, for details). In this paper we present an updated and homogeneous pulsational scenario for a wide range of stellar parameters typical of BL Her stars. In an accompanying paper (Di Criscienzo et al. 2006) we will discuss the connection between the pulsational scenario and the predictions of updated evolutionary models to compare theory with observations.

The organization of the present paper is the following: in Sect. 2 we present the new computed models. In Sect. 3 we deal with predicted light/velocity curves and visual amplitudes. Some important relations are given in Sect. 4 and the conclusions close the paper.

\section{The stellar pulsation models}

To study BL Her stars, we have adopted the same pulsation code and the same physical and numerical assumptions used for RR Lyrae (Bono \& Stellingwerf 1994; Bono et al. 2000; Marconi et al. 2003; Di Criscienzo et al. 2004) and Anomalous Cepheid models (Marconi et al. 2006). Model sequences have been computed as one parameter families with constant luminosity, mass, and chemical composition, by varying the effective temperature in steps of $100 \mathrm{~K}$. The various assumptions of mass, luminosity, 
Table 1. Input parameters of the computed BL Her models. A helium abundance $Y=0.24$ has been adopted.

\begin{tabular}{ccccccc}
\hline \hline$Z$ & $M / M_{\odot}$ & $\log L / L_{\odot}$ & FOBE & FBE & FORE & FRE \\
\hline 0.0001 & 0.60 & 1.95 & - & 6850 & - & 5750 \\
& & 2.05 & - & 6750 & - & 5650 \\
& & 2.15 & - & 6750 & - & 5550 \\
& 0.65 & 1.91 & 6950 & 6850 & 6100 & 5750 \\
& & 2.01 & 6750 & 6850 & 6300 & 5750 \\
& & 2.11 & - & 6750 & - & 5550 \\
\hline 0.001 & 0.50 & 2.11 & - & 6650 & - & 5450 \\
& & 2.41 & - & 6350 & - & 5150 \\
& 0.55 & 1.81 & 6875 & 6850 & 6400 & 5650 \\
& & 1.91 & - & 6850 & - & 5550 \\
& & 2.01 & - & 6750 & - & 5450 \\
& \multirow{2}{*}{0.65} & 1.81 & 7050 & 6750 & 6650 & 5750 \\
& & 1.91 & 6850 & 6750 & 6150 & 5650 \\
& & 2.01 & 6650 & 6850 & 6350 & 5650 \\
\hline 0.004 & 0.55 & 1.81 & - & 6950 & - & 5750 \\
& & 1.91 & - & 6850 & - & 5650 \\
& & 2.01 & - & 6750 & - & 5450 \\
\hline
\end{tabular}

and metallicity are consistent with the most recent evolutionary prescriptions (see Pietrinferni et al. 2004, 2006) and are reported in the first three columns of Table 1.

Linear regression through the model's parameters allows us to derive analytical relations connecting the period of models to the intrinsic stellar parameters, namely mass, luminosity and effective temperature, i.e.:

$$
\begin{aligned}
\log P= & 11.579( \pm 0.015)+0.893 \log \frac{L}{L_{\odot}}-0.89 \log \frac{M}{M_{\odot}} \\
& -3.54 \log T_{\text {eff }}
\end{aligned}
$$

for fundamental pulsators and:

$$
\begin{aligned}
\log P= & 10.784( \pm 0.003)+0.806 \log \frac{L}{L_{\odot}}-0.66 \log \frac{M}{M_{\odot}} \\
& -3.31 \log T_{\text {eff }}
\end{aligned}
$$

for $\mathrm{FO}$ ones.

It is well known that radial pulsation occurs in a quite welldefined region of the HR diagram, the so called instability strip, which depends on the intrinsic parameters of pulsators. For each given metallicity, mass, and luminosity, we have calculated the maximum and minimum effective temperatures for the onset of either fundamental or first-overtone pulsation, as reported in the last four columns of Table 1, where FOBE (FORE) and FBE (FRE) are the blue (red) limit for the first overtone and fundamental pulsator, respectively. It is of importance to note that our calculations confirm the earlier suggestion by Bono et al. (1997a) that, for a given helium content and mass, there exists an "intersection" luminosity $L_{\mathrm{IP}}$ where $\mathrm{FOBE}=\mathrm{FBE}$ and that above this luminosity only the fundamental mode has a stable nonlinear limit cycle. As shown by Di Criscienzo et al. (2006), the evolutionary models show that BL Her variables occur at a luminosity higher than this intersection point: therefore, in the following we will consider only fundamental models. As for the red boundary of the fundamental mode, it is worth mentioning that it is expected to depend on the efficiency of convection and, in particular, on the value of the mixing length parameter $l / H_{\mathrm{p}}$ we assume to close the nonlinear system of dynamical and convective equations (see Stellingwerf 1982; and Bono \& Stellingwerf 1992, 1994, for details on the treatment of convection in the adopted hydrodynamical code). To test this

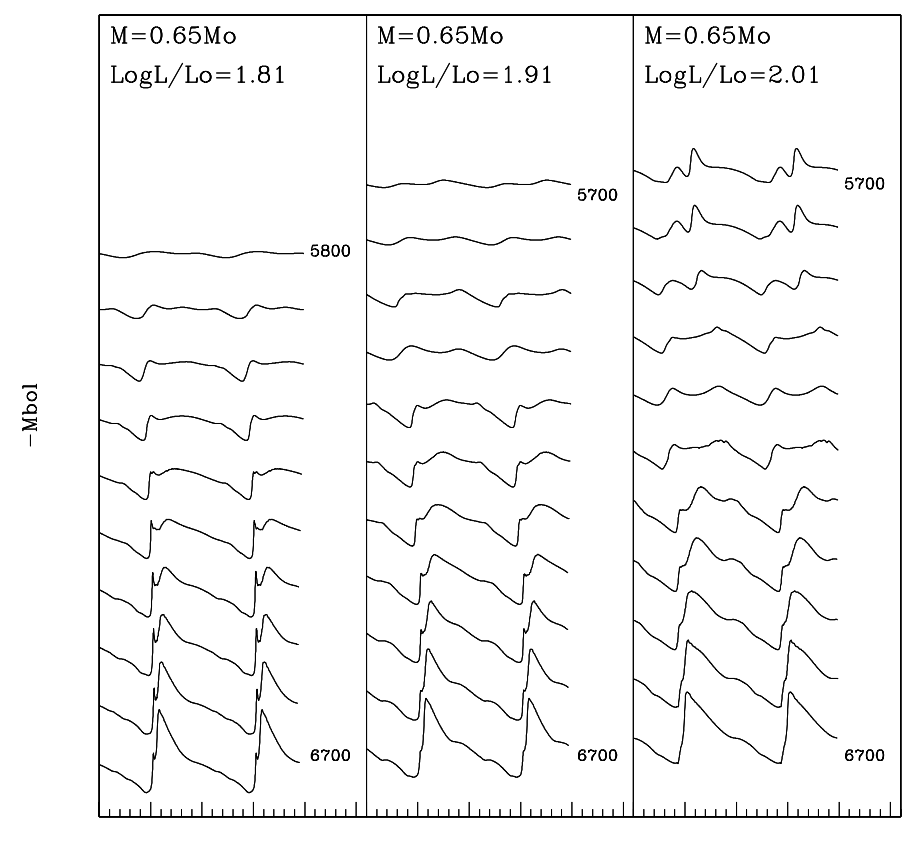

Phase

Fig. 1. Theoretical bolometric light curves for a subsample $(Z=0.001$ and $M=0.65 M_{\odot}$ ) of fundamental models. The luminosity levels are labeled.

occurrence, we have computed additional models with $l / H_{\mathrm{p}}$ increased from 1.5 to 2.0 and we confirm the general trend shown by RR Lyrae (Di Criscienzo et al. 2004) and Classical Cepheid models (Fiorentino et al. 2006, in preparation), in that the FRE moves toward higher effective temperatures as $l / H_{\mathrm{p}}$ increases. In addition, we find that this effect is less important with increasing the luminosity. In particular, for $M=0.50 M_{\odot}$ and $Z=0.001$ the FRE blueshift is of about $200 \mathrm{~K}$ at $\log L / L_{\odot}=2.01$, but reduces to less than $100 \mathrm{~K}$ for $\log L / L_{\odot}=2.41$. This occurrence is due to the reduced density values, and in turn to the increase of the required superadiabaticity, in the outer layers of stellar structures with low mass and very high luminosity.

\section{Light curves and pulsation amplitudes}

For each investigated model, the non-linear analysis provides the variation of relevant parameters, namely luminosity, radius, radial velocity, effective temperature, and gravity, along a pulsational cycle. A subsample of bolometric light curves $(Z=$ 0.001 and $M=0.65 M_{\odot}$ and the labeled luminosity levels) is shown in Fig. 1, while Fig. 2 exhibits the corresponding radial velocity curves as a function of pulsation phase. All these curves show a large variety of shapes, which is perhaps the most striking feature of BL Her models, as already suggested by Moskalik \& Buchler (1994). In all the sequences (in particular for radial velocity curves), the presence and the progression of the main bump is evident in analogy to what is observed for Classical Cepheids with periods around 10 days (the well-known Hertzsprung progression). A detailed investigation of this issue, through the study of Fourier parameters, will be addressed in a future paper.

To compare theoretical results with observations, the bolometric lights curves have been transformed into the photometric bands $U B V R I J K$, using bolometric corrections and temperaturecolor relations provided by Castelli et al. (1997a,b). In this way, light-curve amplitudes and mean absolute magnitudes, either 


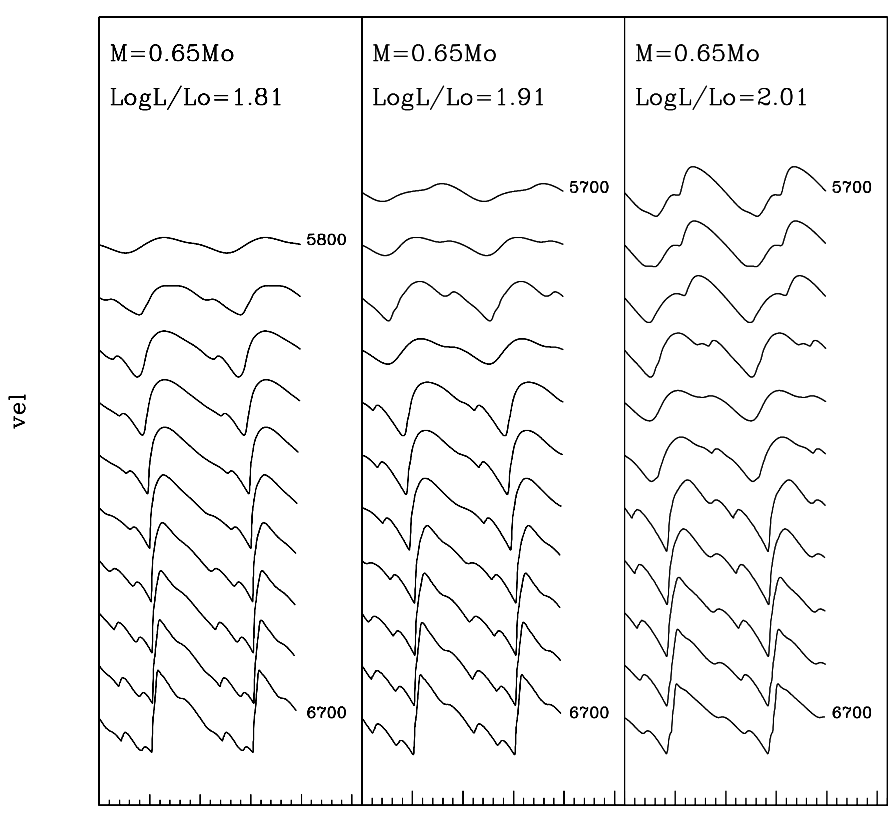

Phase

Fig. 2. The same as Fig. 2, but for theoretical radial velocity curves.

intensity-weighted $\langle M\rangle$ or magnitude-weighted $(M)$, are derived in various photometric bands. After having tested that intensityweighted magnitudes approximate the static values better than magnitude-weighted ones, similarly to what found for RR Lyrae (see, e.g., Marconi et al. 2003) and Classical and Anomalous Cepheids (Caputo et al. 1999; Marconi et al. 2004), in the following we give the predicted relations with $\langle M\rangle$ also because empirical investigations usually provide this type of mean value $^{1}$

In Fig. 3, we show the behavior of visual amplitudes as a function of the period for different values of metallicity, mass, and luminosity. As for RR Lyrae models, we find that for a fixed period the amplitude increases as the luminosity increases and as the mass decreases (middle and bottom left panels, respectively), while it remains quite constant in the considered interval of metallicity, at fixed mass and luminosity (top left panel). We notice that part of the observed behavior in the period-amplitude diagram is due to the dependence of period on mass and luminosity, as simply derived even from a simple linear adiabatic approach. The change in the pulsation in amplitude is mainly related to the distance from the FBE, as shown in the two right panels of the same figure. The deviation from linearity of the highest luminosity level models (top panel of Fig. 3) is related to the complex coupling between pulsation and convection for these low density and cool structures (see also Bono et al. 1997 b for a similar behavior in high luminosity RR Lyrae stars). However, in the range of linearity we obtain:

$\log P=-0.033( \pm 0.034)-1.15 \log \frac{M}{M_{\odot}}-0.475 \log \left\langle M_{V}\right\rangle$ $-0.195 A_{V}$

We notice that this dependence of the period on amplitude is, within the errors, in agreement with those found in Di Criscienzo et al. (2004).

${ }^{1}$ Similar relations involving magnitude-weighted mean values are available upon request to the authors.

\section{Same relevant relations}

The linear regression through the data reported in Table 1 provides the following analytical relations:

$$
\begin{aligned}
& \log T_{\mathrm{e}}(F B E)=3.912( \pm 0.005)-0.035 \log \frac{L}{L_{\odot}}+0.048 \log \frac{M}{M_{\odot}} \\
& \log T_{\mathrm{e}}(F R E)=3.925( \pm 0.005)-0.075 \log \frac{L}{L_{\odot}}+0.118 \log \frac{M}{M_{\odot}}
\end{aligned}
$$

with an rms of 0.005 , valid on the adopted range of metallicity and stellar mass. The location of these edges in the $M_{V}-\log P$ plane is presented in Fig. 4 and the corresponding analytical relations are:

$$
\begin{aligned}
\left\langle M_{V}(F B E)\right\rangle= & -0.36( \pm 0.04)-1.55 \log \frac{M}{M_{\odot}}-2.37 \log P \\
& +0.05( \pm 0.03) \log Z \\
\left\langle M_{V}(F R E)\right\rangle= & 0.14( \pm 0.03)-2.25 \log \frac{M}{M_{\odot}}-2.17 \log P \\
& +0.05( \pm 0.02) \log Z .
\end{aligned}
$$

From the same figure, we note that the BL Her instability strip is the extension at higher luminosities of the fundamental instability region for RR Lyrae stars, whereas it does not overlap the one for anomalous cepheids (ACs) since the AC FRE is hotter than the BL Her FBE (see also Caputo et al. 2004). This result, also related to the significant mass difference between the two classes of pulsators, is fully consistent with the empirical evidence that ACs are brighter than BL Her stars, for a given period.

The natural outcome of the period relation (Eq. (1)) in the observational plane is the Period-Magnitude-Color relation in which the pulsation period is correlated with the pulsator absolute magnitude and color, for a given mass. As an example, linear interpolations through the results give:

$$
\begin{aligned}
\left\langle M_{V}\right\rangle= & -1.22( \pm 0.05)-2.60 \log P-3.03\left[\left\langle M_{B}\right\rangle-\left\langle M_{V}\right\rangle\right] \\
& -1.10 \log \frac{M}{M_{\odot}} \\
\left\langle M_{V}\right\rangle= & -2.08( \pm 0.02)-2.88 \log P-4.13\left[\left\langle M_{V}\right\rangle-\left\langle M_{K}\right\rangle\right] \\
& -2.14 \log \frac{M}{M_{\odot}}
\end{aligned}
$$

with no dependence on metallicity. According to these relations, for a sample of variables at the same distance and with the same reddening, e.g., for variables in a given globular cluster or galaxy with no differential reddening, one could estimate the mass range spanned by the variables from the observed luminosity range, once periods and intrinsic colors are firmly known. However, these relations have the disadvantage of depending on reddening uncertainties and for this reason, as discussed in several earlier papers (e.g., Madore 1982; Di Criscienzo et al. 2004; Udalski et al. 1999), Period-Wesenheit relations are widely used, with the Wesenheit functions being by definition reddening insensitive. The full set of the predicted Period-Wesenheit and PeriodMagnitude relations, including the evolutionary properties of the BL Her stars, are reported in Di Criscienzo et al. (2006). Figure 5 shows the behavior of the predicted radii for fundamental pulsators, obtained by averaging the theoretical radius curve. The solid line is the linear regression through the entire set of models $(P R$ relation):

$\log R=0.87( \pm 0.01)+0.529( \pm 0.006) \log P$,

where $R$ is in solar units. This relation is in agreement with the empirical $P R$ relation obtained by Burki \& Meylan (1986). 


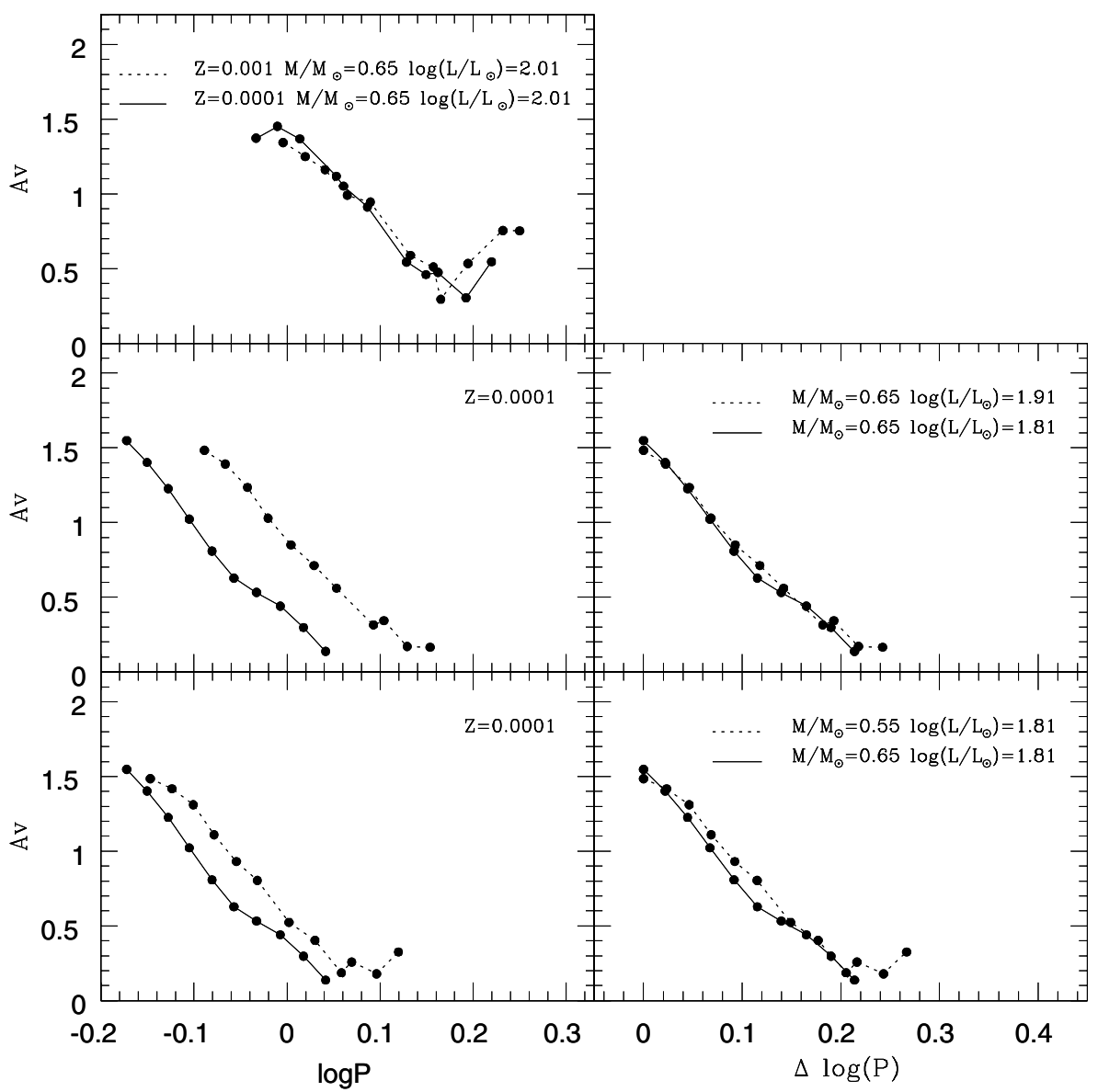

Fig. 3. (Right panels) Visual amplitudes versus periods for selected models varying the metallicity at fixed mass and luminosity (upper panel), varying the luminosity at fixed mass and metallicity $(Z=0.001$, intermediate panel $)$ and varying the mass at fixed luminosity and metallicity $(Z=0.001$, lower panel). The same but visual amplitudes are now plotted versus $\Delta \log (P)=\log (P)-\log \left(P_{\mathrm{FOBE}}\right)$ (left panels).

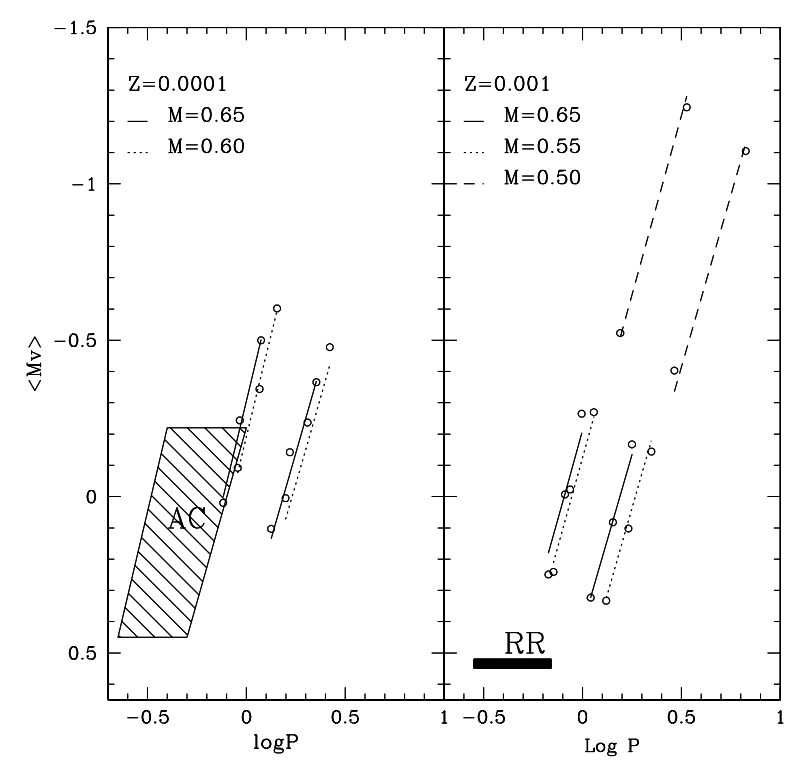

Fig. 4. Dependence of the boundaries of the pulsation region on luminosity in the $M_{V}-\log P$ plane for the labeled values of mass and metallicity. In the left panel we also report the AC instability strip for a mass of 1.3 $M_{\odot}$ (Marconi et al. 2004), while in the right one we show the predicted RR Lyrae instability strip for $Z=0.001$ as obtained by synthetic simulations.

In the same figure we have also reported the theoretical $P R$ relation recently obtained by Marconi et al. (2005) for RR Lyrae. This very good agreement between the theoretical

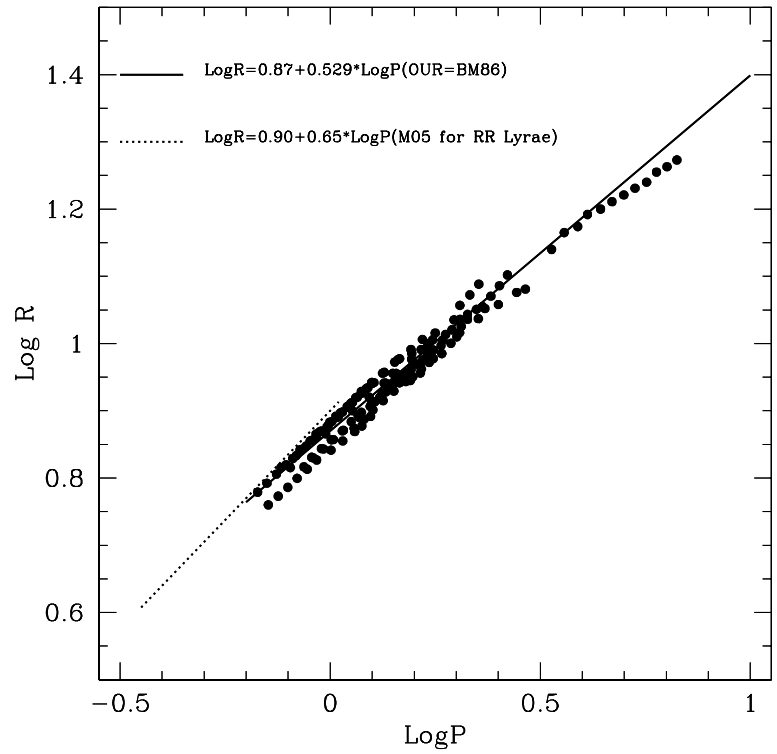

Fig. 5. Period-Radius relation for our models. The solid line is the linear regression throught the entire set of BL Her models (filled circles), which is exactly the same as that found by Burki \& Meylan (1996, BM96), while the dashed one is the PR relation obtained by Marconi et al. (2005, M05) for RR Lyrae stars.

PR relations obtained for RR Lyrae and BL Her stars supports earlier suggestions by Burki \& Meylan (1986) concerning the similarity of the $P R$ relations for these two classes of Pop. II 
variables, and is consistent with the results by Caputo et al. (2004) concerning the behavior of RR Lyrae and BL Her in the Period-Magnitude diagram.

\section{Conclusion and final remarks}

This work is part of a larger project that has the scope to study, from the theoretical point of view, Population II variables in globular clusters and dwarf galaxies. In the last few years we have provided the results for RR Lyrae stars (Marconi et al. 2003; Di Criscienzo et al. 2004) and anomalous cepheids (Marconi et al. 2004; Caputo et al. 2004; Fiorentino et al. 2006). The present paper, together with the investigation by Di Criscienzo et al. (2006), deals with a further class of radial pulsators observed in similar metal poor stellar fields: Population II Cepheids. In particular, we present the new nonlinear and convective pulsational models for short-period variables, generally named BL Her stars.

We have investigated the instability strip and shown the dependence of the pulsation region on mass and luminosity, as well as the effect of varying the mixing lenght parameter. We have presented a detailed atlas of light/radial-velocity curves. All these curves show a large variety of shapes, which is perhaps the most striking feature of BL Her stars, as already suggested by Moskalik \& Buchler (1994) on the basis of non-linear but radiative models.

Theoretical light curves have been transformed in the UBVRIJK photometric bands, using the bolometric corrections and color-temperature relations provided by Castelli et al. (1997a,b). On this basis, mean magnitudes and colors have been evaluated and some relations both for the boundaries of the instability strip and for the dependence of the absolute magnitude on period, mass, and color are derived. Finally, we have calculated the period-radius relation and found that it is in excellent agreement with the empirical relation by Burki \& Meylan (1996) and is consistent with the one holding for shorter periods for RR Lyrae stars.

Acknowledgements. It is a pleasure to thank F. Caputo for useful discussions and for her critical reading of the early draft of this paper. Financial support for this study was provided by MIUR, under the scientif project "On the evolution of stellar systems: a fundamental step toword the scientific exploitation of "VST" (P.I Massimo Capaccioli).

\section{References}

Alcock, C., Allsman, R., Alves, D., et al. 1998, AJ, 115, 192 Bono, G., \& Stellingwerf, R. F. 1994, ApJS, 93, 233

Bono, G., Caputo, F., \& Santolamazza, P. 1997a, A\&A, 317, 171

Bono, G., Caputo, F., Castellani, V., \& Marconi, M. 1997b, A\&AS, 121, 327 (BLU)

Bono, G., Castellani, V., \& Marconi, M. 2000a, ApJ, 532, L129

Bono, G., Marconi, M., \& Stellingwerf, R. F. 2000b, ApJ, 532, 129

Buchler, J. R., \& Moskalik, P. 1994, A\&A, 292, 450

Buchler, J. R., \& Buchler, N. E. G. 1994, A\&A, 285, 213

Burki, G., \& Meylan, G. 1986, A\&A, 159, 255

Cacciari, C., \& Clementini, G. 2003, LNP, 635, 105

Castelli, F., Gratton, R. G., \& Kurucz, R. L. 1997a, A\&A, 318, 841

Castelli, F., Gratton, R. G., \& Kurucz, R. L. 1997b, A\&A, 324, 432

Caputo, F. 1998, A\&ARv, 9, 33

Caputo, F., Castellani, V., Marconi, M., \& Ripepi, V. 1999, MNRAS, 306, 815

Caputo, F., Castellani, V., Degl'Innocenti, S., Fiorentino, G., \& Marconi, M. 2004, A\&A, 424, 927

Cardelli, J. A., Clayton, G. C., \& Mathis, J. S. 1989, ApJ, 345, 245

Di Criscienzo, M., Marconi, M., \& Caputo, F. 2004, ApJ, 612, 1092

Di Criscienzo, M., Caputo, F., Marconi, M., \& Musella, I. 2005, MNRAS, in press [arXiv: astro-ph/0511128]

Di Criscienzo, M., Caputo, F., Marconi, M., \& Cassisi, S. 2006, A\&A, submitted (D06)

Fiorentino, G., Marconi, M., \& Musella, I. 2006, in preparation

Fiorentino, G., Marconi, M., Limongi, M., \& Caputo, F., A\&A, in press

Gingold, R. A. 1985, MmSAI, 56, 169

Kubiak, M., \& Udalski, A. 2003, AcA, 53, 117

Harris, H. C. 1985, AJ, 90, 756

Madore, B. F. 1982, ApJ, 253, 575

Marconi, M., Caputo, F., Di Criscienzo, M., \& Castellani, M. 2003, ApJ, 596, 299

Marconi, M., Fiorentino, G., \& Caputo, F. 2004, A\&A, 417, 1101

Moskalik, P., \& Buchler, J. R. 1993, ApJ, 406, 190

Nemec, J. M., Nemec, A. F. L., \& Lutz, T. E. 1994, AJ, 108, 222

Sandage, A., \& Tammann 2006, ARA\&A, 44, 93

Sandage, A., Diethelm, R., \& Tammann, G. 1994, A\&A, 283, 111

Udalski, A., Soszynski, I., Szymanski, M., et al. 1999, AcA, 49, 223

Wallerstein, G. 1990, ASPC, 11, 56

Wallerstein, G. 2002, PASP, 114, 689

Wallerstein, G., \& Cox, A. N. 1984, PASP, 96, 677 